\title{
In-silico Molecular Docking and ADME/Pharmacokinetic Prediction Studies of Some Novel Carboxamide Derivatives as Anti-tubercular Agents
}

\author{
Mustapha Abdullahi ${ }^{1}$ (I) Shola Elijah Adeniji ${ }^{1}$ (1)
}

Received: 16 April 2020 / Accepted: 3 July 2020 / Published online: 10 July 2020

(c) The Author(s) 2020

\begin{abstract}
Molecular docking simulation of thirty-five (35) molecules of $N$-(2-phenoxy)ethyl imidazo[1,2-a]pyridine-3-carboxamide (IPA) with Mycobacterium tuberculosis target (DNA gyrase) was carried out so as to evaluate their theoretical binding affinities. The chemical structure of the molecules was accurately drawn using ChemDraw Ultra software, then optimized at density functional theory (DFT) using Becke's three-parameter Lee-Yang-Parr hybrid functional (B3LYP/6-311**) basis set in a vacuum of Spartan 14 software. Subsequently, the docking operation was carried out using PyRx virtual screening software. Molecule 35 (M35) with the highest binding affinity of $-7.2 \mathrm{kcal} / \mathrm{mol}$ was selected as the lead molecule for structural modification which led to the development of four (4) newly hypothetical molecules D1, D2, D3 and D4. In addition, the D4 molecule with the highest binding affinity value of $-9.4 \mathrm{kcal} / \mathrm{mol}$ formed more H-bond interactions signifying better orientation of the ligand in the binding site compared to M35 and isoniazid standard drug. In-silico ADME and drug-likeness prediction of the molecules showed good pharmacokinetic properties having high gastrointestinal absorption, orally bioavailable, and less toxic. The outcome of the present research strengthens the relevance of these compounds as promising lead candidates for the treatment of multidrug-resistant tuberculosis which could help the medicinal chemists and pharmaceutical professionals in further designing and synthesis of more potent drug candidates. Moreover, the research also encouraged the in vivo and in vitro evaluation study for the proposed designed compounds to validate the computational findings.
\end{abstract}

Keywords Molecular docking $\cdot$ Binding affinity $\cdot$ Active sites $\cdot$ Pharmacokinetics $\cdot$ Molecular interactions $\cdot$ Hydrogen bond

\section{Introduction}

Mycobacterium tuberculosis (MTB) is the microorganism that most frequently affects lungs causing one of the most deadly respiratory and communicable diseases worldwide called tuberculosis (TB) [1]. It can be spread from one person to another through the air, but it is preventable and more importantly curable $[2,3]$. The World Health Organization (WHO) established that a total of 1.5 million TB infected patients died in 2018 (including 251,000 HIV/

Mustapha Abdullahi

mustychem19@gmail.com

Shola Elijah Adeniji

shola4343@gmail.com

1 Department of Chemistry, Faculty of Physical Sciences, Ahmadu Bello University, PMB 1044, Zaria, Kaduna, Federal Republic of Nigeria
AID infected patients). Besides, more than $87 \%$ new cases of TB were reported in the 30 countries with high TB burden in which 8 countries accounted for the two-thirds of the total with India on the lead, followed by China, Indonesia, the Philippines, Pakistan, Nigeria, Bangladesh, and South Africa [4]. Furthermore, multidrug-resistant TB (MDR-TB) remains a public health and security crisis. There are more than 484,000 new cases reported with resistance to rifampicin (the first-line most-effective drug) of which 78\% had MDR-TB. The WHO estimated that about 58 million saved lives from TB cases through treatment and diagnosis from 2000 to 2018, and also targeting the ending of the TB epidemic by 2030 [4]. The existence of extensively drug-resistant (XDR) and the evolution of multidrug-resistant MDR-TB have attracted the attention of drug scientists who are in search of novel anti-tubercular agents with improving bioactivities [5]. Previous research has shown that imidazo[1,2-a]pyridine-3-carboxamide (IPA) as an anti-tubercular candidate is currently 
in the second phase of clinical trials, and it was reported to have resilient inhibitory potency or anti-mycobacterial activity [5], but no sufficient information on their binding affinity or ligand-receptor molecular interactions. The M. tuberculosis DNA gyrase is an essential nanomachine for the regulation of DNA topology and the only type II topoisomerase present for quinolone action against multidrug-resistant tuberculosis [6]. However, it was established that the drug hunt or development of a more potent molecule with improved bioactivities is very costly and timeconsuming [7]. The concept of computational chemistry such as computer-aided drug design (CADD) might save the time of discovering new molecules which also reduces the cost of synthesis [7, 8]. Molecular docking is one of the CADD technique which predicts the binding affinity as well as the best binding pose of the molecule with the active site of a target (receptor), and it is crucial when carrying out a structure-based drug design (SBDD) [9]. Virtual docking rationalizes sighting new lead compounds, and also provides a hunch on structure-activity relationships (SARs) and mode(s) of activity action based on scoring function and further binding modes analysis from ligand-protein binding/interaction studies [10]. In-silico physicochemical properties of a molecule such as saturation, lipophilicity, polarity, size, solubility, and flexibility give vital information on whether the molecule can serve as a drug at an early stage of development [9]. The aim of this study was to perform virtual screening of thirtyfive (35) molecules of $N$-(2-phenoxy)ethyl imidazo[1,2- $a$ ] pyridine-3-carboxamide (IPA) through molecular docking strategy with $M$. tuberculosis DNA gyrase as the receptor, and identify a possible lead molecule as a template to design new hypothetical molecules with improved binding affinities, and better molecular residual interactions with the receptor. Furthermore, in-silico Absorption, distribution, metabolism, and excretion (ADME) and druglikeness properties of the molecules were also evaluated.

\section{Methodology}

\subsection{Computer System and Software}

Computer system (Dell), with the following specification properties; CPU Dual@0.30 GHz, Intel ${ }^{\circledR}$ Core i3-6100U, 12 Gigabyte RAM was used throughout the present study. The software download and installed include PyRx virtual screening software, Spartan'14V 1.1.4, Discovery Studio Visualizer v16.1.0.15350, Chemdraw Ultra software V. 12.0.2, Spartan'14V 1.1.4 developed by Wave function Inc., Swiss ADME online software.

\subsection{In-Silico Docking And ADME/Pharmacokinetics Prediction}

Thirty-five (35) molecules were taken from the newly synthesized analogs of some $N$-(2-phenoxy)ethyl imidazo[1,2a]pyridine-3-carboxamides (IPAs) as anti-tubercular agents [5]. The compound's response against the $M$. tuberculosis (MTB-H37Rv) was measured as minimum inhibitory concentration (MIC) which were converted into logarithmic MIC values (pMIC) using Eq. (1).

$p M I C=-\log \left(\frac{M I C(\mu \mathrm{g} / \mathrm{mL})}{M w(\mathrm{~g} / \mathrm{mol})} \times 10^{-3}\right)$,

where $\mathrm{Mw}$ is the molar weight of the compound in $\mathrm{g} / \mathrm{mol}$ and MIC is the minimum inhibitory concentration of the compound. The chemical structure of the molecules was accurately drawn with ChemDraw Ultra level software V12.0.2 (Table 1), then optimized with Spartan 14 software at density functional theory (DFT) with Becke's three-parameter Lee-Yang-Parr hybrid functional (B3LYP/6-31G** basis set) in a vacuum [11], which was later saved as Protein Data Bank (PDB) file format. In principle, the geometry optimization using the DFT level of theory as an iterative process for the search of the lowest energy conformer give the most stable conformation of each molecule in the dataset [12]. The M. tuberculosis DNA Gyrase subunit B crystal structure (PDB ID: 3IG0) of H37Rv organism strain was downloaded as the receptor from the PDB website: https://www.rcsb.org at a model quality of $2.1 \AA$ resolution [8]. In-silico docking operation was carried out on the 35 molecules with the downloaded receptor using AutoDock Vina of PyRx virtual screening software [13]. The vina wizard uses a stochastic gradient optimization algorithm for predicting the binding affinities between ligands and receptors. The docking output with the highest binding affinity was visualized to study the residual interactions using Discovery Studio Visualizer. Figure 1 shows the 3D prepared DNA gyrase target and M35 ligand. Furthermore, the Simplified Molecular Input Line Entry System (SMILES) format of the molecules was pasted on the swiss ADME webserver (Swiss Institute of Bioinformatics, Switzerland) to generate their ADME/pharmacokinetic profile and drug-likeness parameters.

\section{Results and Discussions}

\subsection{Molecular Docking and Virtual Screening}

Molecular docking is a simulation technique that explores ligand's best binding pose with the active site of a target [14]. This technique involves the selection of 3D- coordinate 
Table 1 Molecular binding affinities of the imidazo[1,2- $a$ ]pyridine3-carboxamide (IPA) analogs as anti-MTB agents with DNA Gyrase receptor (PDB ID: 3IG0)

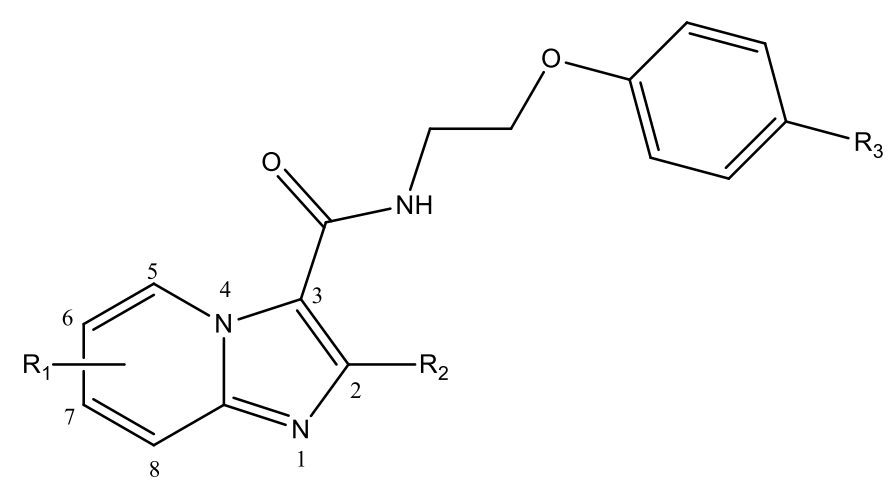

IPA core structure

\begin{tabular}{|c|c|c|c|c|c|c|}
\hline Compound ID & $\mathrm{R}_{1}$ & $\mathrm{R}_{2}$ & $\mathrm{R}_{3}$ & $\mathrm{MIC}(\mu \mathrm{g} / \mathrm{mol})$ & pMIC & $\begin{array}{l}\text { Binding } \\
\text { scores (kcal/ } \\
\text { mol) }\end{array}$ \\
\hline M1 & $6-\mathrm{NO}_{2}$ & $\mathrm{Me}$ & $\mathrm{Br}$ & 1.28 & 5.5150 & -6.8 \\
\hline M2 & $6-\mathrm{F}$ & $\mathrm{Me}$ & $\mathrm{Br}$ & 1.208 & 5.5112 & -6.6 \\
\hline M3 & $6-\mathrm{Cl}$ & $\mathrm{Me}$ & $\mathrm{Br}$ & 0.488 & 5.9220 & -6.7 \\
\hline M4 & $6-\mathrm{Br}$ & $\mathrm{Me}$ & $\mathrm{Br}$ & 0.358 & 6.1012 & -6.2 \\
\hline M5 & 6-OMe & $\mathrm{Me}$ & $\mathrm{Br}$ & 0.028 & 7.1592 & -6.5 \\
\hline M6 & 7-Me & $\mathrm{Me}$ & $\mathrm{Br}$ & 0.463 & 5.9233 & -6.4 \\
\hline M7 & 7-Cl & $\mathrm{Me}$ & $\mathrm{Br}$ & 2.682 & 5.1822 & -6.7 \\
\hline M8 & 8-Me & $\mathrm{Me}$ & $\mathrm{Br}$ & 13.925 & 4.4451 & -6.4 \\
\hline M9 & $8-\mathrm{Cl}$ & $\mathrm{Me}$ & $\mathrm{Br}$ & 0.505 & 5.9073 & -6.2 \\
\hline M10 & $5-\mathrm{Cl}$ & $\mathrm{Me}$ & $\mathrm{Br}$ & 2.524 & 5.2085 & -6.3 \\
\hline M11 & $6-\mathrm{Cl}$ & Et & $\mathrm{Br}$ & 0.023 & 7.2636 & -6.5 \\
\hline M12 & $6-\mathrm{F}$ & Et & $\mathrm{Br}$ & 0.202 & 6.3032 & -6.4 \\
\hline M13 & $6-\mathrm{Br}$ & Et & $\mathrm{Br}$ & 0.025 & 7.2704 & -6.7 \\
\hline M14 & 7-Cl & Et & $\mathrm{Br}$ & 6.354 & 4.8222 & -6.5 \\
\hline M15 & $8-\mathrm{Cl}$ & Et & $\mathrm{Br}$ & 17.524 & 4.3817 & -6.2 \\
\hline M16 & 6-Me & Et & $\mathrm{Br}$ & 0.275 & 6.1649 & -6.8 \\
\hline M17 & 6-Me & $\mathrm{n}-\operatorname{Pr}$ & $\mathrm{Br}$ & 0.415 & 6.0011 & -6.2 \\
\hline M18 & 6-Me & $\mathrm{c}-\mathrm{Pr}$ & $\mathrm{Br}$ & 0.052 & 6.9010 & -6.5 \\
\hline M19 & $6-\mathrm{F}$ & $n-P r$ & $\mathrm{Br}$ & 2.682 & 5.1948 & -6.1 \\
\hline M20 & $6-\mathrm{F}$ & c-Pr & $\mathrm{Br}$ & 0.928 & 5.6536 & -6.4 \\
\hline M21 & $6-\mathrm{Cl}$ & $n-P r$ & $\mathrm{Br}$ & 0.054 & 6.9071 & -5.6 \\
\hline M22 & $6-\mathrm{Cl}$ & $\mathrm{c}-\mathrm{Pr}$ & $\mathrm{Br}$ & 0.098 & 6.6462 & -6.3 \\
\hline M23 & 7-Cl & $\mathrm{c}-\mathrm{Pr}$ & $\mathrm{Br}$ & 32 & 4.1323 & -5.9 \\
\hline M24 & 7-Me & $\mathrm{c}-\mathrm{Pr}$ & $\mathrm{Br}$ & 5.004 & 4.9177 & -6.6 \\
\hline M25 & 6-Me & $n-P r$ & $\mathrm{Cl}$ & 0.631 & 5.7706 & -6.2 \\
\hline M26 & $6-\mathrm{F}$ & $\mathrm{Me}$ & $\mathrm{Cl}$ & 3.841 & 4.9572 & -7.0 \\
\hline M27 & 7-Cl & Et & $\mathrm{Cl}$ & 5.862 & 4.8095 & -7.0 \\
\hline M28 & $6-\mathrm{Cl}$ & Et & $\mathrm{Cl}$ & 0.029 & 7.1151 & -6.6 \\
\hline M29 & $6-\mathrm{Cl}$ & $n-P r$ & $\mathrm{Cl}$ & 0.631 & 5.7933 & -6.1 \\
\hline M30 & $6-\mathrm{F}$ & $n-P r$ & $\mathrm{Cl}$ & 1.141 & 5.5180 & -5.9 \\
\hline M31 & 7-Cl & $\mathrm{c}-\mathrm{Pr}$ & $\mathrm{Cl}$ & 32 & 4.0859 & -6.1 \\
\hline M32 & $6-\mathrm{Cl}$ & Et & $\mathrm{OMe}$ & 0.056 & 6.8248 & -5.8 \\
\hline M33 & 6-Me & $\mathrm{c}-\mathrm{Pr}$ & $\mathrm{OMe}$ & 0.027 & 7.1323 & -6.1 \\
\hline
\end{tabular}


Table 1 (continued)

\begin{tabular}{|c|c|c|c|c|c|c|}
\hline Compound ID & $\mathrm{R}_{1}$ & $\mathrm{R}_{2}$ & $\mathrm{R}_{3}$ & $\mathrm{MIC}(\mu \mathrm{g} / \mathrm{mol})$ & pMIC & $\begin{array}{l}\text { Binding } \\
\text { scores (kcal/ } \\
\text { mol) }\end{array}$ \\
\hline M34 & 6-Me & Et & $\mathrm{OMe}$ & 0.023 & 7.1874 & -5.9 \\
\hline M35 & $6-\mathrm{Br}$ & Et & -5 & 0.0625 & 6.9219 & -7.2 \\
\hline IND & - & - & - & 0.049 & 7.3098 & -4.8 \\
\hline
\end{tabular}

IND isoniazid

Fig. 1 3D prepared DNA gyrase receptor (PDB: 3IG0) and lead molecule 35 (M35)
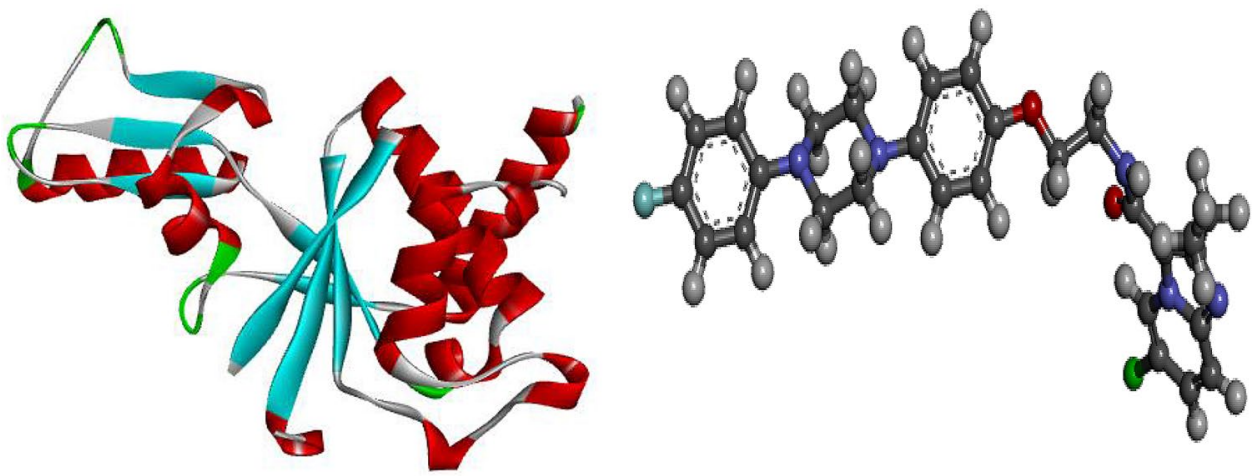

space of the binding site in the target and calculating the binding affinity of the resultant orientation of the molecule within the binding site which forms the complex [14]. The significance and sensitivity of binding affinity values are determined by the largest magnitude negative number (highest binding affinity or lowest binding energy) depicting the most favourable conformation of the complex formed when the ligand involved efficiently binds with the active pockets of the target [15]. In addition, the molecular docking simulation was performed to validate the anti-tubercular efficacy of synthesized imidazo [1,2- $a$ ] pyridine-3-carboxamide derivatives by investigating binding modes as well as orientation of ligands in the receptor pocket of MTB-DNA gyrase target. The docking poses were ranked according to their score values, and Table 1 showed binding affinities of the best pose of the selected 35 synthesized molecules with the MTB-DNA gyrase target. It was observed that the binding affinity of complexes ranged from -5.8 to $-7.2 \mathrm{kcal} / \mathrm{mol}$ which confirmed their excellent potency. Also, the docking results are in agreement with their anti-MTB activities as reported by Wang et al. [5]. Thus, M35 molecule as the best inhibitor with the highest binding affinity of $-7.2 \mathrm{kcal} /$ mol was selected as the lead molecule (template) in which further structural alterations were made to explore new hypothetical molecules. The design was done in such a way that the synthesis of the hypothetical compounds would be experimentally feasible. Furthermore, the designing strategy was guided by introducing some active pharmacophores in isoniazid (standard drug), and haloalkyl fragment (trifluoromethyl) to the template. As such, four (4) novel designed analogs such as 6-chloro-2-ethyl- $N$-(2-((5-(4-(4-fluorophenyl)piperazin-1-yl)-4-(hydrazinecarbonyl)pyridin-2-yl)oxy) ethyl)imidazo[1,2-a]pyridine-3-carboxamide (D1), 6-chloro1-ethyl- $N$-(((2-(4-(hydrazinecarbonyl)-3-(trifluoromethyl) pyridin-1(2H)-yl)-3-oxo-3,4-dihydro-2 $H$-pyrido[1,2-a]pyrazin-7-yl)oxy)methyl)imidazo[1,5-a]pyridine-7-carboxamide (D2), 6-chloro-1-ethyl- $N$-((4-(4-(4-fluorophenyl)-2-oxo-3,4dihydropyrazin-1(2H)-yl)phenoxy)methyl)imidazo[1,5-a] pyridine-7-carboxamide (D3), 6-chloro-1-ethyl- $\mathrm{N}$-(((2(3-fluoro-4-(hydrazinecarbonyl)pyridin-1(2H)-yl)-3-oxo3,4-dihydro-2H-pyrido[1,2-a]pyrazin-7-yl)oxy)methyl) imidazo[1,5- $a$ ]pyridine-7-carboxamide (D4) with their binding affinities of $-8.0 \mathrm{kcal} / \mathrm{mol},-8.3 \mathrm{kcal} / \mathrm{mol},-8.5 \mathrm{kcal} /$ mol and $-9.4 \mathrm{kcal} / \mathrm{mol}$ were reported (Fig. 2) to have improved binding affinity with the receptor compared to the M35 molecule. Moreover, the molecules in this study have better binding affinity than isoniazid standard drug (known inhibitor) with a binding affinity of $-4.8 \mathrm{kcal} / \mathrm{mol}$.

M35 molecule (template) formed six (6) hydrophobic interactions with 4 amino acid residues (Ala 644, Ala 533, Pro 566, and Leu 568) in the active pocket of the target having no any hydrogen bond as shown in Fig. 3. The chloro 
<smiles>CCc1nc2ccc(Cl)cn2c1C(=O)NCCOc1ccc(N2CCN(c3ccc(F)cc3)CC2)cc1</smiles>

M35 (-7.2 kcal/mol)<smiles>CCc1nc2ccc(Cl)cn2c1C(=O)NCCOc1cc(C(=O)NN)c(N2CCN(c3ccc(F)cc3)CC2)cn1</smiles>

D1 (-8.0 kcal $/ \mathrm{mol})$<smiles>CCc1ncn2cc(Cl)c(C(=O)NCOc3ccc(N4C=CN(c5ccc(F)cc5)CC4=O)cc3)cc12</smiles>

D3 $(-8.5 \mathrm{kcal} / \mathrm{mol})$<smiles>NNC(=O)c1ccncc1</smiles>

Isoniazid (-4.8 kcal/mol)<smiles>CCc1ncn2cc(Cl)c(C(=O)NCOC3=CN4CC(=O)N(N5C=CC(C(=O)NN)=C(C(F)(F)F)C5)C=C4C=C3)cc12</smiles>

D2 (-8.3 kcal/mol)

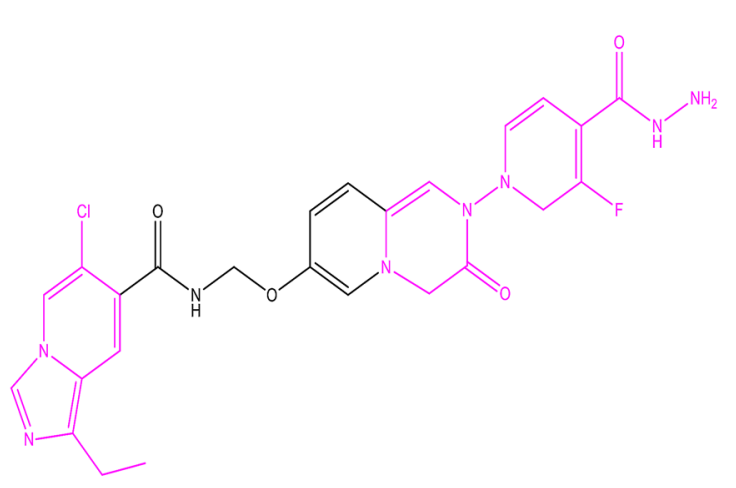

D4 (-9.4 kcal/mol)

Fig. 2 The 2D structures of the designed IPA compounds from M35 molecule (template) and their binding affinity (kcal/mol)

Fig. 3 Docking interaction of complex 35 (M35)

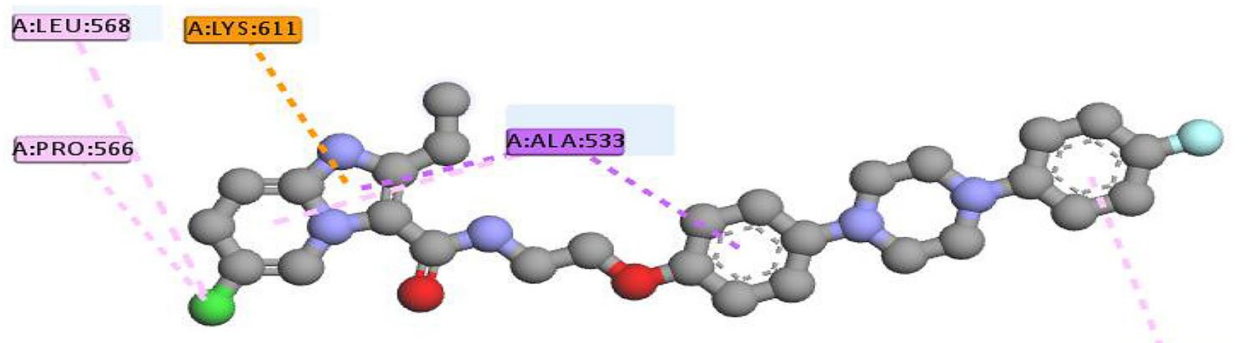

A:ALA:644

Interactions

Pi-Cation

Pi-Sigma 
substituent $(-\mathrm{Cl})$ attached to imidazo[1,2- $a$ ]pyridine fragment formed two (2) hydrophobic interactions (Pi-Sigma) with Pro 566 and Leu 568 at different distances of $4.00 \AA$ and $5.32 \AA$, while the delocalized $\Pi$-electron of the benzene rings and imidazole moiety in the molecule also interact to form another hydrophobic interactions with amino acid residues of Ala 533 and Ala 644 at different distances of $3.82 \AA$, $3.52 \AA, 3.44 \AA$ and $5.48 \AA$ respectively. Other noticeable interaction is the electrostatic with Lys 611 at $4.15 \AA$ due to Pi-Cation interactions. Isoniazid is a known inhibitor (standard drug) interacts with the active pocket of the receptor (PDB ID: 3IG0) to form three (3) H-bonds with Arg 550, Phe 549 and His 514 residues at bond distances of 2.13, 2.78 and $2.90 \AA$ respectively. Other interactions includes one (1) hydrophobic bond with Pro 554 (4.90 A) and unfavorable positive-positive interaction with Arg 553 at $4.208 \AA$ as interaction distance as elucidated in Fig. $4 a$, b.

D1 molecule was designed by adding acetohydrazide fragment to the pyridine ring of the M35 at the C4 position. Therefore, the D1 molecule formed two (2) H-bond interactions with Glu 586 and Phe 580 at bond distances of $2.24 \AA$ and $2.09 \AA$ respectively. Also, D1 formed three (3) hydrophobic interactions (Pi-Alkyl and Alkyl) with amino acid residues of Val 632, Phe 580, and Pro 578 at different bond distances of $4.10 \AA, 4.89 \AA$ and $5.21 \AA$ accordingly. Other interactions include one (1) C-H bond with Pro 578 (3.26 $\AA$ ) and three (3) Pi-anion interactions (electrostatic) with Glu 623, Asp 577 and Glu 586 at $3.44 \AA$, $4.33 \AA$ and $3.64 \AA$ as interaction distance elucidated in Fig. $4 \mathrm{a}$, b. Based on the information obtained from D1, we rearranged the 6-chloro-2-ethylimidazo[1,2-a]pyridine fragment of the template (M35) to 6-chloro-1-ethylimidazo[1,5-a]
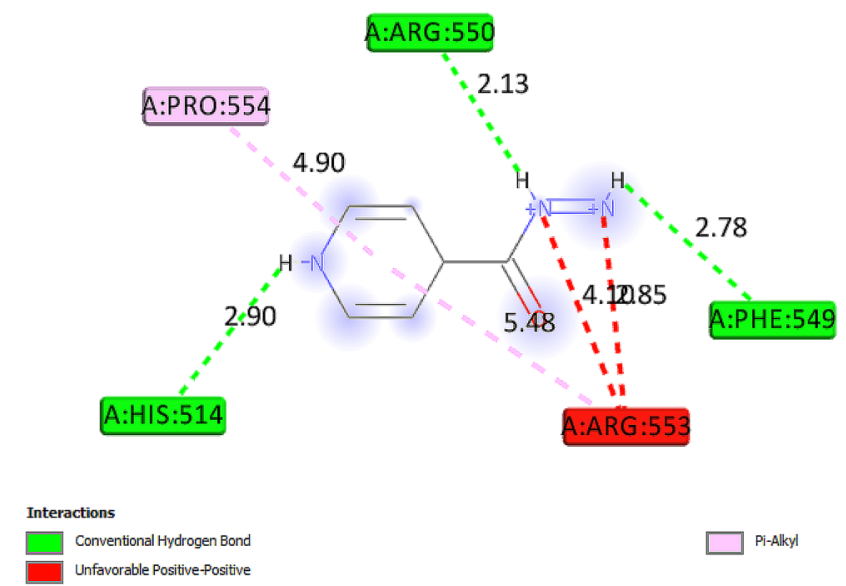

\section{(A) 2D interactions of Isoniazid with the receptor}

Fig. 4 a, b 2D and 3D docking interaction of isoniazid pyridine and further addition of functional fragments like acetohydrazide, trifluoromethyl led to D2, D3 and D4 as shown in Fig. 2.

D2 molecule formed three (3) hydrogen bonds where the $-\mathrm{NH}$ of acetohydrazide and fluoro of trifluoromethyl $\left(-\mathrm{CF}_{3}\right)$ substituent strongly interact with Leu 529 and Ser 541 at $2.65 \AA, 2.18 \AA$ and $2.75 \AA$ distances respectively. D2 also formed six (6) hydrophobic interactions such as Pi-sigma, Pi-Alkyl, Alkyl with Ala 533 (3.70, 4.18 \& $4.55 \AA$ A), Pro 566 and Leu 568 (4.9 ̊ \& $5.07 \AA$ ), and Leu 563 (5.41 $\mathrm{\AA}$ ). Other noticeable interaction includes electrostatic interaction with Lys 611 at $4.23 \AA$ and a C-H bond with Ser 541 at $3.46 \AA$ distance. Similarly, the D3 molecule also formed one (1) a Conventional H-bond with Glu 586 at a distance of $2.04 \AA$ An addition, D3 formed three (3) hydrophobic such as Alkyl and Pi-Alkyl interactions with amino acid residues of Val 632 (5.37 $\AA$ and $4.77 \AA$ ), and Val 630 at $4.34 \AA$ distances. Other interactions formed by the D3 include electrostatic type (Pi-anion interactions) with Glu 586, Glu 623 and Asp 572 at distances of 3.53, 3.91 and $3.71 \AA$ respectively, two (2) C-H bond with Pro 578 and Glu 623 at 3.50 and $3.70 \AA$ distances. D4 molecule formed four (4) conventional H-bonds with Gln 538, Arg 634, Ala 531, Lys 611 at $2.32 \AA, 2.57 \AA, 2.19 \AA$ and $2.31 \AA$ respectively. Furthermore, D4 also formed four (4) hydrophobic interactions (Pi-Sigma and Alkyl) with Ala 533 (3.47 and $4.37 \AA$ ), Pro 566 at $4.45 \AA$, and Leu 568 at $5.41 \AA$ as bond distances. Numerous molecular docking studies have reported that the number of hydrogen bonds and distances are the key factors influencing the binding affinity of a ligand-receptor interaction [16]. As such, this reason gives a structural insight as to

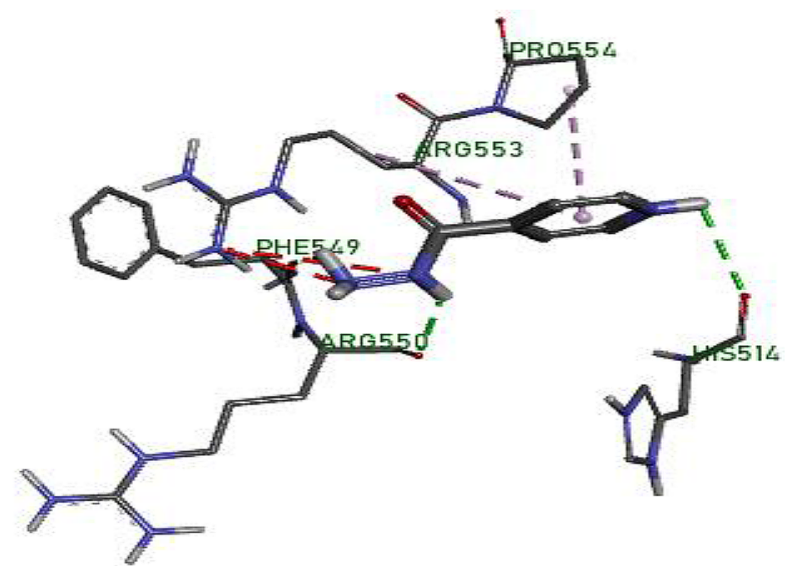


why the designed molecule D4 was able to bind tightly with the active pocket of the target enzyme.

The docking interaction of the complex D1, D2, D3, and D4 were respectively shown in Figs. 5, 6, 7 and 8 accordingly.

\subsection{In-silico ADME/Pharmacokinetic Predictions}

It was established that the antagonistic response of inhibitors with an enzyme or a protein receptor cannot promise the suitability of an inhibitor as a potential drug [15]. Therefore, ADME (absorption, distribution, metabolism, and excretion) including drug-likeness analysis are important in the drug discovery which helps to make a rational decision on whether inhibitors can be administered to a biological system or not $[10,15]$. In addition, inhibitors with poor ADME properties and high toxicity effects on the biological systems are often the major cause of most failed medicines in the clinical phase of experiments. The Pfizer's rule of five also known as Rule of five (Ro5) or Lipinski's rule of five (5) by Christopher A. Lipinski in 1997 is a thumb-rule for evaluating drug-likeness and to decide if an inhibitor with a certain biological and pharmacological properties would be an orally active drug in the human body [10]. The rule states

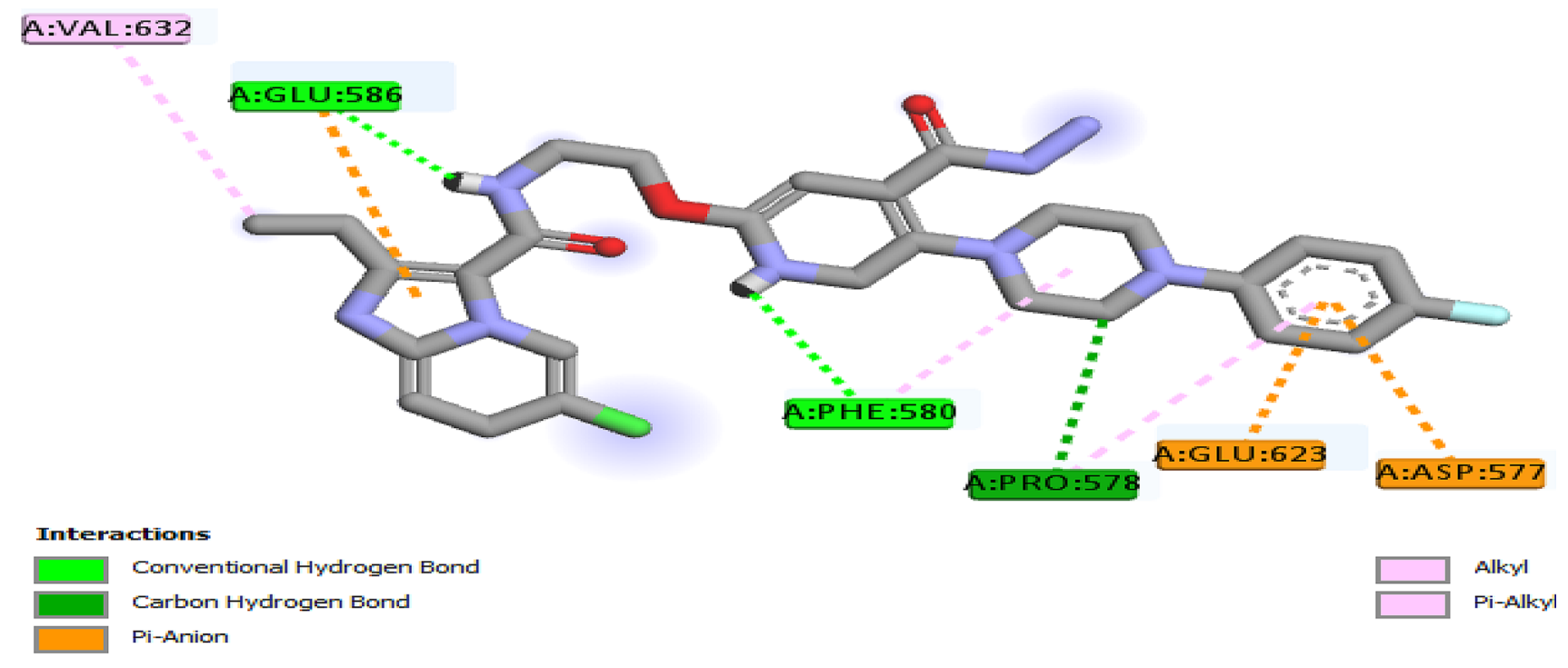

Fig. 5 Docking interaction of complex D1

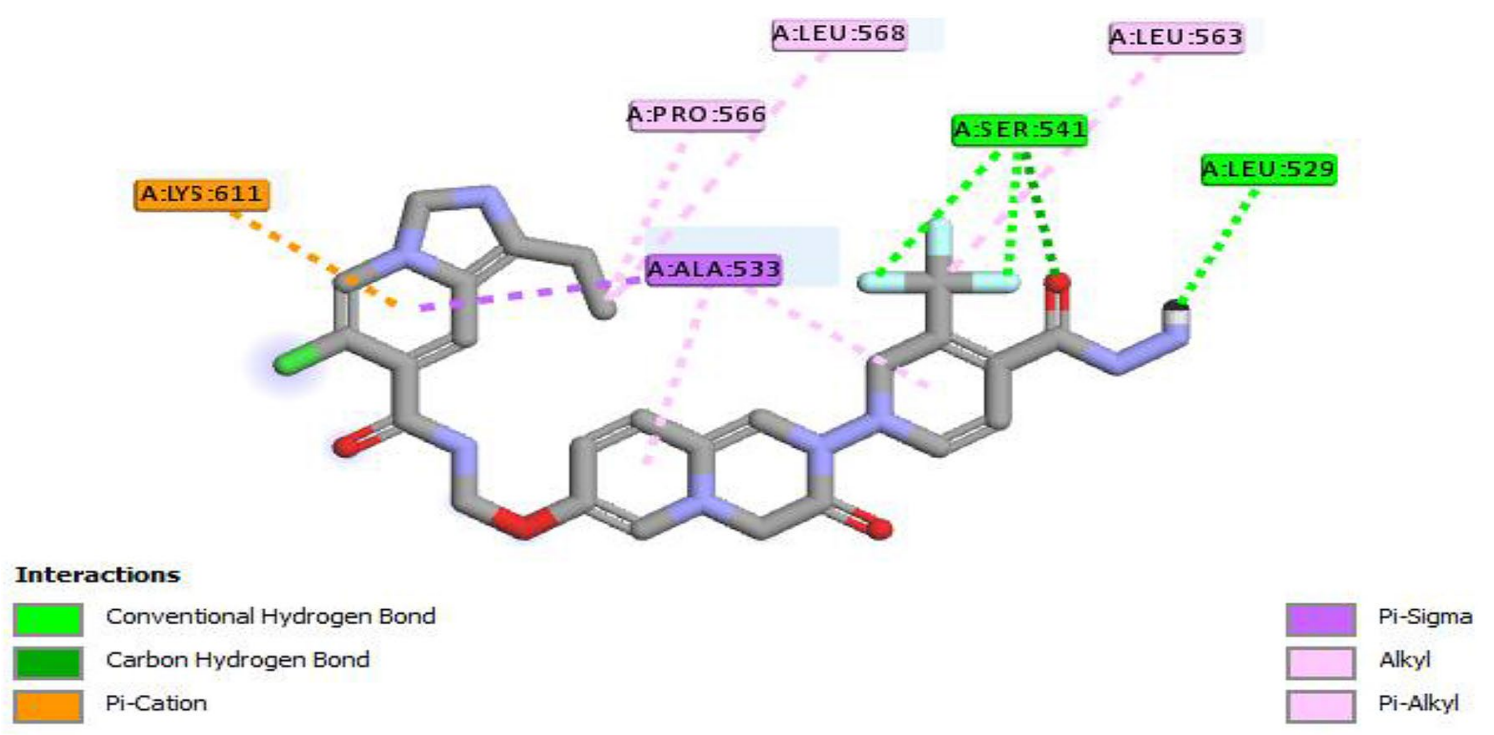

Fig. 6 Docking interaction of complex D2 


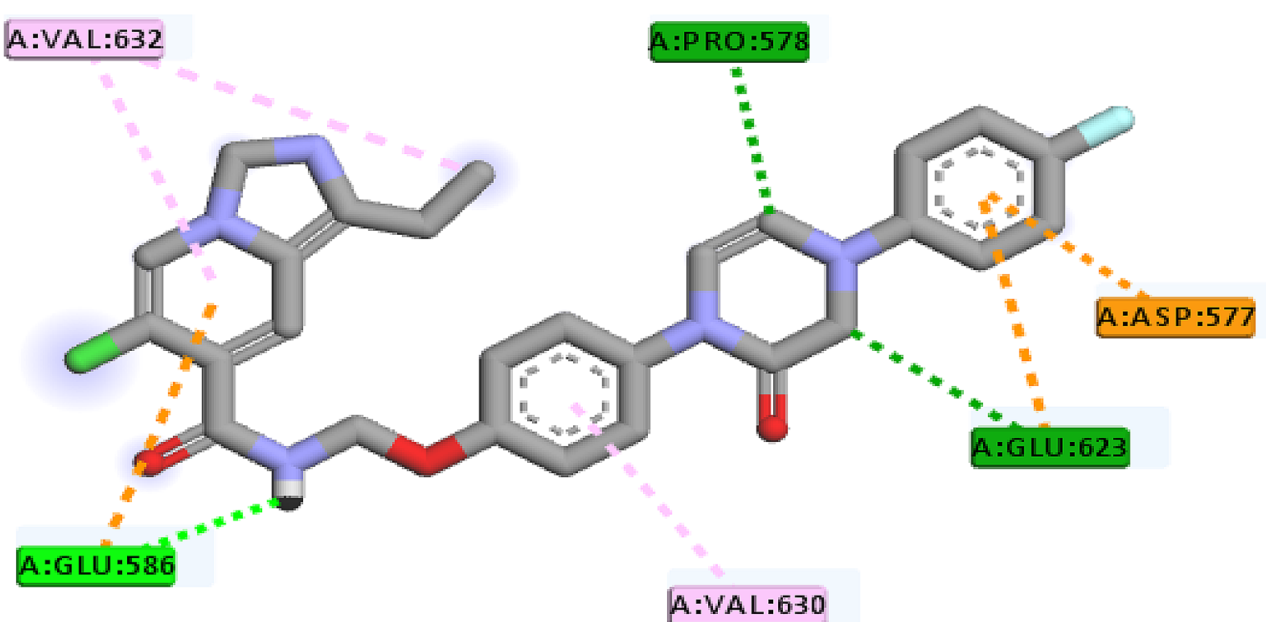

\section{Interactions}

Conventional Hydrogen Bond

Carbon Hydrogen Bond

Pi-Anion

Fig. 7 Docking interaction of complex D3

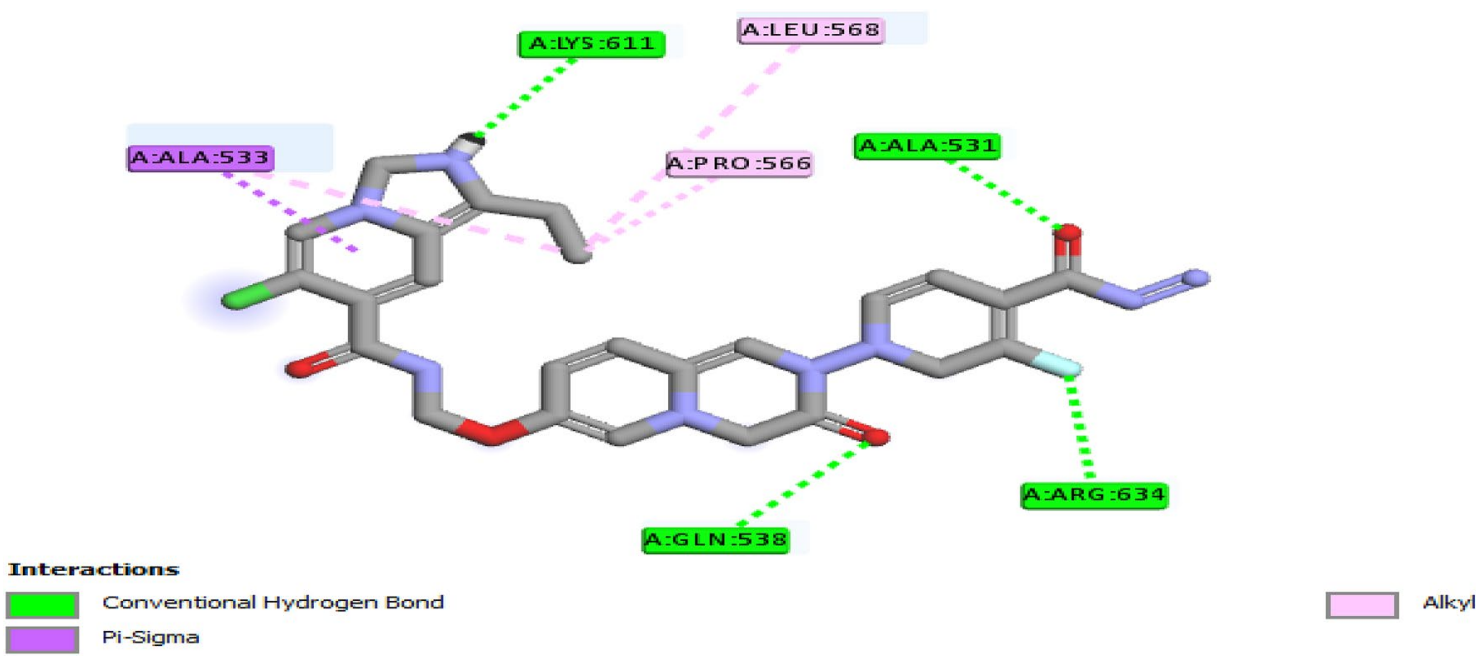

Fig. 8 Docking interaction of complex D4

that a molecule or an inhibitor can be orally absorbed/active if two (2) or more of these thresholds; molecular weight (Mw) of molecule $<500$, octanol/water partition coefficient $(\mathrm{iLOGP}) \leq 5$, number of hydrogen bond acceptors $(\mathrm{nHBA}) \leq 10$, number of hydrogen bond donors $(\mathrm{nHBD}) \leq 5$, and topological polar surface area (TPSA) $<40 \AA^{2}$ ) are not violated. From the output of some ADME and drug-likeness properties shown in Table 2, it was observed that M1 to M34 molecules have zero violations of the Lipinski's rule, and M35 violated only the molecular weight rule with $522.01 \mathrm{~g} /$ mol. The drug-likeness parameters are related to aqueous solubility and intestinal permeability which determines the first step of oral bioavailability [17]. The results also showed good pharmacokinetic properties in which all molecules have high gastrointestinal absorption, non- substrate to P-glycoprotein except for (M35), and only M1 possess blood-brain barrier BBB permeant. The Brain Or IntestinaL EstimateD permeation predictive model (BOILED-Egg), also known as Egan egg graph (Fig. 9) of the 35 molecules was also generated from SwissADME online webserver which revealed a clear graphical representation of the absorption of the molecules in the brain and gastrointestinal tract [18]. The graph molecules in the yolk area (yellow) are predicted to inactively permeate the blood-brain barrier 
Table 2 ADME and druglikeness parameters of the IPA molecules

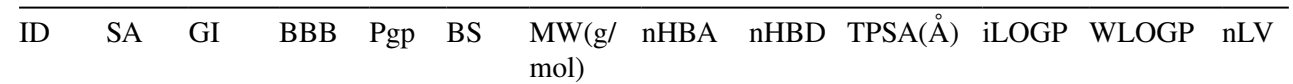

$\mathrm{mol})$

\begin{tabular}{|c|c|c|c|c|c|c|c|c|c|c|c|c|}
\hline M1 & 2.78 & High & No & No & 0.55 & 419.23 & 5 & 1 & 101.45 & 3.1 & 3.12 & 0 \\
\hline M2 & 2.62 & High & Yes & No & 0.55 & 392.22 & 4 & 1 & 55.63 & 3.46 & 3.77 & 0 \\
\hline M3 & 2.59 & High & Yes & No & 0.55 & 408.68 & 3 & 1 & 55.63 & 3.4 & 3.87 & 0 \\
\hline M4 & 2.59 & High & Yes & No & 0.55 & 453.13 & 3 & 1 & 55.63 & 3.45 & 3.98 & 0 \\
\hline M5 & 2.85 & High & Yes & No & 0.55 & 404.26 & 4 & 1 & 64.86 & 3.53 & 3.22 & 0 \\
\hline M6 & 2.6 & High & Yes & No & 0.55 & 388.26 & 3 & 1 & 55.63 & 3.56 & 3.52 & 0 \\
\hline M7 & 2.52 & High & Yes & No & 0.55 & 408.68 & 3 & 1 & 55.63 & 3.76 & 3.87 & 0 \\
\hline M8 & 2.62 & High & Yes & No & 0.55 & 388.26 & 3 & 1 & 55.63 & 3.59 & 3.52 & 0 \\
\hline M9 & 2.59 & High & Yes & No & 0.55 & 408.68 & 3 & 1 & 55.63 & 3.52 & 3.87 & 0 \\
\hline M10 & 2.67 & High & Yes & No & 0.55 & 408.68 & 3 & 1 & 55.63 & 3.34 & 3.87 & 0 \\
\hline M11 & 2.78 & High & Yes & No & 0.55 & 422.7 & 3 & 1 & 55.63 & 3.62 & 4.12 & 0 \\
\hline M12 & 2.8 & High & Yes & No & 0.55 & 406.25 & 4 & 1 & 55.63 & 3.18 & 4.03 & 0 \\
\hline M13 & 2.77 & High & Yes & No & 0.55 & 467.15 & 3 & 1 & 55.63 & 3.68 & 4.23 & 0 \\
\hline M14 & 2.72 & High & Yes & No & 0.55 & 422.7 & 3 & 1 & 55.63 & 3.98 & 4.12 & 0 \\
\hline M15 & 2.79 & High & Yes & No & 0.55 & 422.7 & 3 & 1 & 55.63 & 3.69 & 4.12 & 0 \\
\hline M16 & 2.81 & High & Yes & No & 0.55 & 402.28 & 3 & 1 & 55.63 & 3.82 & 3.78 & 0 \\
\hline M17 & 3 & High & Yes & No & 0.55 & 416.31 & 3 & 1 & 55.63 & 3.9 & 4.17 & 0 \\
\hline M18 & 2.93 & High & Yes & No & 0.55 & 414.3 & 3 & 1 & 55.63 & 3.9 & 4.03 & 0 \\
\hline M19 & 2.98 & High & Yes & No & 0.55 & 420.28 & 4 & 1 & 55.63 & 3.65 & 4.42 & 0 \\
\hline M20 & 2.91 & High & Yes & No & 0.55 & 418.26 & 4 & 1 & 55.63 & 3.69 & 4.28 & 0 \\
\hline M21 & 2.95 & High & Yes & No & 0.55 & 436.73 & 3 & 1 & 55.63 & 3.87 & 4.51 & 0 \\
\hline M22 & 2.89 & High & Yes & No & 0.55 & 434.71 & 3 & 1 & 55.63 & 3.84 & 4.37 & 0 \\
\hline M23 & 2.84 & High & Yes & No & 0.55 & 434.71 & 3 & 1 & 55.63 & 3.87 & 4.37 & 0 \\
\hline M24 & 2.93 & High & Yes & No & 0.55 & 414.3 & 3 & 1 & 55.63 & 4.34 & 4.03 & 0 \\
\hline M25 & 2.97 & High & Yes & No & 0.55 & 371.86 & 3 & 1 & 55.63 & 3.76 & 4.06 & 0 \\
\hline M26 & 2.59 & High & Yes & No & 0.55 & 347.77 & 4 & 1 & 55.63 & 3.54 & 3.66 & 0 \\
\hline M27 & 2.74 & High & Yes & No & 0.55 & 378.25 & 3 & 1 & 55.63 & 3.37 & 4.01 & 0 \\
\hline M28 & 2.93 & High & Yes & No & 0.55 & 392.28 & 3 & 1 & 55.63 & 3.61 & 4.4 & 0 \\
\hline M29 & 2.95 & High & Yes & No & 0.55 & 375.82 & 4 & 1 & 55.63 & 3.47 & 4.31 & 0 \\
\hline M30 & 2.81 & High & Yes & No & 0.55 & 390.26 & 3 & 1 & 55.63 & 3.83 & 4.26 & 0 \\
\hline M31 & 2.81 & High & Yes & No & 0.55 & 390.26 & 3 & 1 & 55.63 & 3.83 & 4.26 & 0 \\
\hline M32 & 2.89 & High & Yes & No & 0.55 & 373.83 & 4 & 1 & 64.86 & 3.75 & 3.37 & 0 \\
\hline M33 & 3.05 & High & Yes & No & 0.55 & 365.43 & 4 & 1 & 64.86 & 3.8 & 3.27 & 0 \\
\hline M34 & 2.92 & High & Yes & No & 0.55 & 353.41 & 4 & 1 & 64.86 & 3.79 & 3.02 & 0 \\
\hline M35 & 3.65 & High & Yes & Yes & 0.55 & 522.01 & 4 & 1 & 62.11 & 4.56 & 4.48 & 1 \\
\hline
\end{tabular}

$S A$ synthetic accessibility, GI gastrointestinal absorption, $B B B$ blood-brain barrier permeant, Pgp P-glycoprotein substrate, $M W$ molecular weight, $n H B D$ number of hydrogen bond donor, $n H B A$ number of hydrogen bond acceptor, BS Bioavailability Score, TPSA topological polar surface area, WLOGP water partition coefficient, $n L V$ number of Lipinski violation
(M1-34), while M35 is predicted to be highly absorbed in the gastrointestinal tract. These theoretical findings are in agreement with the experimentally pharmacokinetic profile reported by Wang et al. [5].

Table 3 showed the ADME and drug-likeness properties of the newly designed molecules which predicted that all designed compounds (D1, D2, D3 and D4) are BBB permeant and highly absorbable in the gastrointestinal tract, while D1, D3 and D4 possess P-glycoprotein. Furthermore, the bioavailability radar shows a rapid appraisal of the drug-likeness of a molecule by taking six (6) physicochemical properties into consideration: saturation, lipophilicity, polarity, size, solubility, and flexibility [18]. Figure 10 showed the bioavailability radars of M35 (lead compound), and the four (4) designed hypothetical molecules (D1, D2, D3 and D4). The molecules are predicted to be orally bioavailable (low flexibility and polarity), less toxic, and good absorption. 

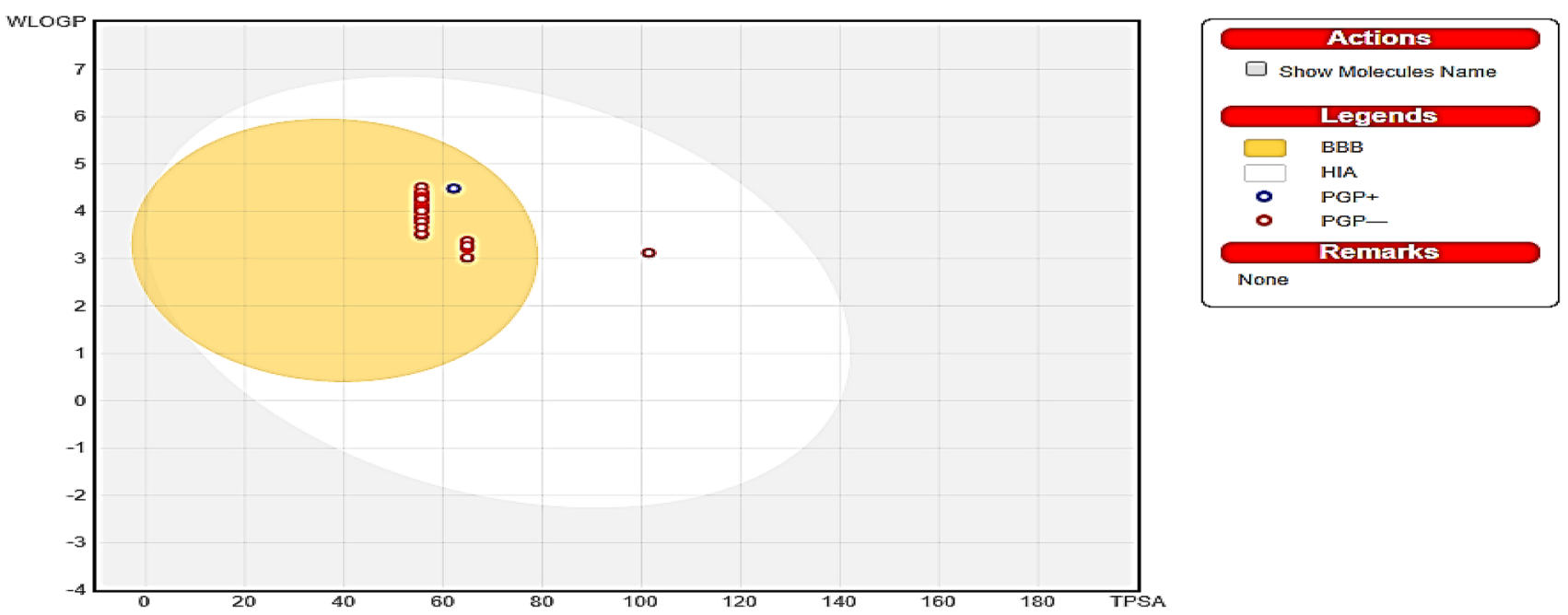

Fig. 9 BOILED-Egg graph of the IPA molecules

Table 3 ADME and druglikeness parameters of the four (4) designed IPA molecules

\begin{tabular}{llllllllllll}
\hline Molecule ID & SA & GI & BBB & Pgp & BS & $\begin{array}{l}\text { MW(g/ } \\
\text { mol) }\end{array}$ & nHBA & nHBD & TPSA(Å) & iLOGP \\
\hline D1 & 4.18 & High & No & Yes & 0.17 & 581.04 & 7 & 3 & 130.12 & 3.81 \\
D2 & 4.78 & High & No & Yes & 0.17 & 604.97 & 9 & 3 & 139.23 & 3.33 \\
D3 & 3.72 & High & No & No & 0.55 & 519.95 & 5 & 1 & 79.18 & 4.14 \\
D4 & 4.71 & High & No & Yes & 0.17 & 554.96 & 7 & 3 & 139.23 & 3.27 \\
\hline
\end{tabular}

\section{Conclusion}

In the present research, the theoretical evaluation of binding affinities $(\mathrm{kcal} / \mathrm{mol})$ of some IPAs with MTB-DNA Gyrase target was carried out in order to validate their potency and to identify a possible lead molecule for structural modifications. The molecular docking results showed a good docking score ranged from $-5.8 \mathrm{kcal} / \mathrm{mol}$ to $-7.2 \mathrm{kcal} / \mathrm{mol}$ signifying that the molecules can bind more tightly with the active site of the target. M35 with the best binding pose was selected as the template for structural modification which led to the development of four (4) newly hypothetical molecules D1, D2, D3 and D4 with the better binding energy of $-8.0 \mathrm{kcal} / \mathrm{mol},-8.3 \mathrm{kcal} /$ $\mathrm{mol},-8.5 \mathrm{kcal} / \mathrm{mol}$ and $-9.4 \mathrm{kcal} / \mathrm{mol}$ respectively when compared with template molecule M35 and the isoniazid standard drug. In addition, the results of the ADME and drug-likeness properties revealed that the 35 molecules and the 4 newly designed molecules have good pharmacokinetic properties which are predicted to be orally bioavailable, less toxic, and good absorption. Furthermore, the increased number of $\mathrm{H}$-bonds formed by the designed compound 4 (D4) having interaction distances less than $3.0 \AA$ gives the structural insight to support the claim why it was able to bind tightly with the active pocket of the targeted enzyme. The outcome of the research strengthens the relevance of these compounds as promising lead(s) for the treatment of multidrug-resistant tuberculosis which could help the medicinal chemists and pharmaceutical professionals in further designing and synthesis of more potent drug candidates. Moreover, the research also encouraged the in vivo and in vitro evaluation study for the proposed designed compounds to validate the computational findings. 


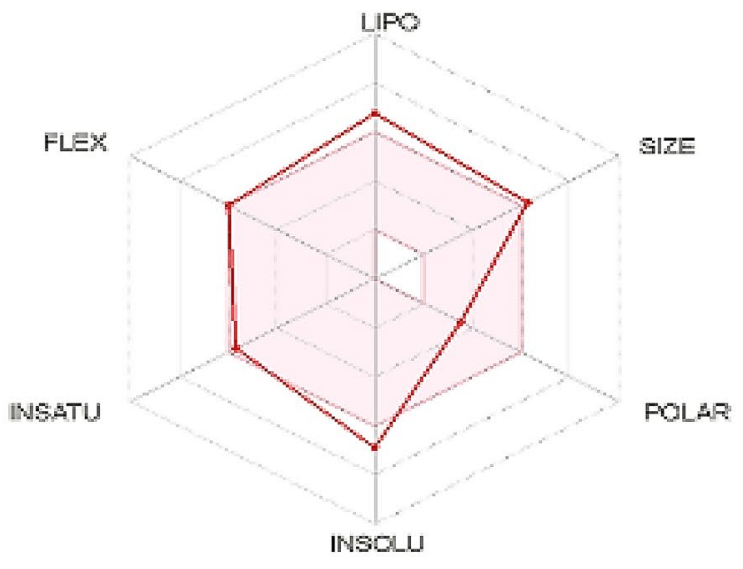

\section{Molecule 35 (template)}

D1

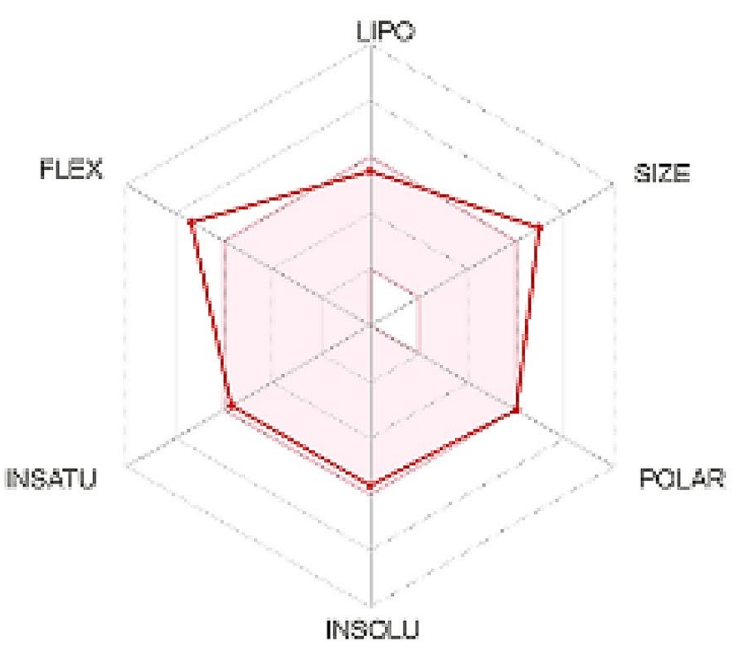

D3

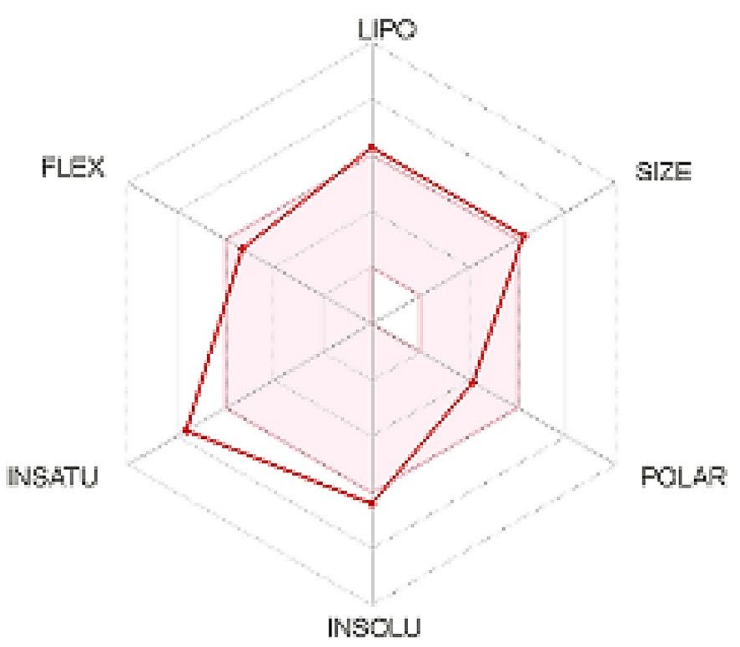

D2

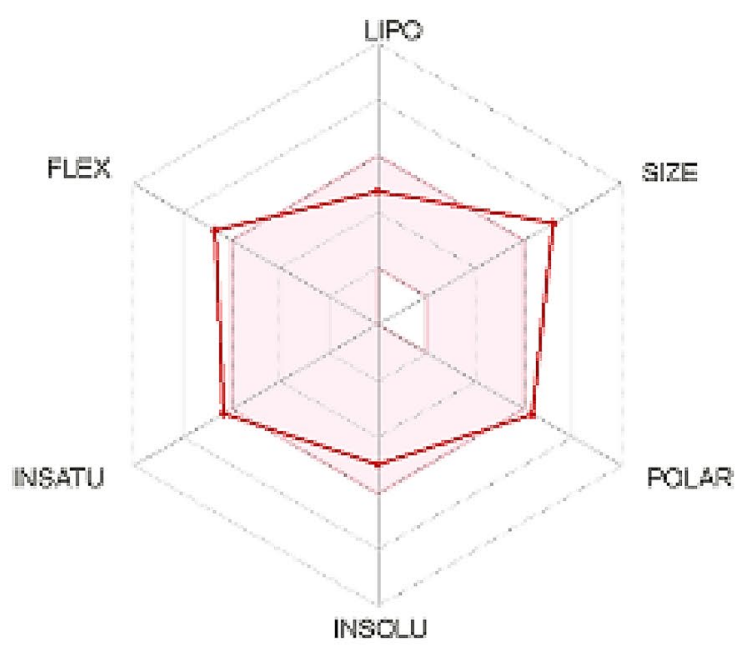

D4

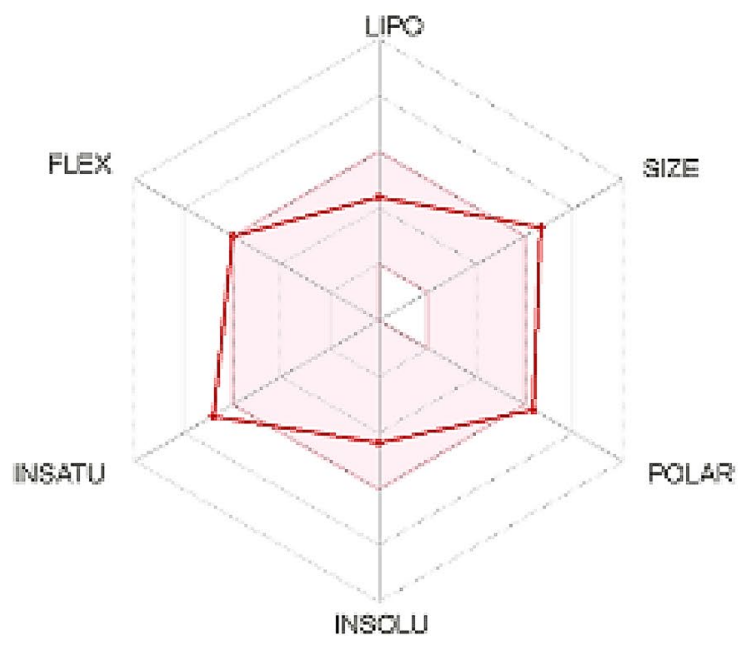

Fig. 10 Bioavailability radar of the lead molecule (M35) and designed molecules 
Acknowledgements The authors expressed their profound gratitude to members of staff in the physical chemistry unit, department of chemistry, Ahmadu Bello University Zaria for their technical assistance.

Open Access This article is licensed under a Creative Commons Attribution 4.0 International License, which permits use, sharing, adaptation, distribution and reproduction in any medium or format, as long as you give appropriate credit to the original author(s) and the source, provide a link to the Creative Commons licence, and indicate if changes were made. The images or other third party material in this article are included in the article's Creative Commons licence, unless indicated otherwise in a credit line to the material. If material is not included in the article's Creative Commons licence and your intended use is not permitted by statutory regulation or exceeds the permitted use, you will need to obtain permission directly from the copyright holder. To view a copy of this licence, visit http://creativecommons.org/licenses/by/4.0/.

\section{References}

1. Zhai W, Wu F, Zhang Y, Fu Y, Liu Z (2019) The immune escape mechanisms of mycobacterium tuberculosis. Int J Mol Sci. https ://doi.org/10.3390/ijms20020340

2. Mabhula A, Singh V (2019) Drug-resistance in mycobacterium tuberculosis: where we stand. MedChemComm 10(8):1342-1360. https://doi.org/10.1039/c9md00057g

3. Ogbuabor DC, Onwujekwe OE (2019) Governance of tuberculosis control programme in Nigeria. Infect Dis Poverty 8(1):1-11. https ://doi.org/10.1186/s40249-019-0556-2

4. World Health Organization (WHO) (2019) Global tuberculosis report-executive summary, Geneva

5. Wang A, Lv K, Li L et al (2019) Design, synthesis and biological activity of $N$-(2-phenoxy)ethyl imidazo[1,2-a]pyridine-3-carboxamides as new antitubercular agents. Eur J Med Chem 178:715725. https://doi.org/10.1016/j.ejmech.2019.06.038

6. Aubry A, Mayer C (2010) Structural insights into the quinolone resistance mechanism of mycobacterium tuberculosis DNA gyrase. PLoS ONE 5(8). https://doi.org/10.1371/journ al.pone. 0012245

7. Abdullahi M, Shallangwa GA, Uzairu A (2020) In silico QSAR and molecular docking simulation of some novel aryl sulfonamide derivatives as inhibitors of $\mathrm{H} 5 \mathrm{~N} 1$ influenza A virus subtype. BeniSuef Univ J Basic Appl Sci 2(9):1-12. https://doi.org/10.1186/ s43088-019-0023-y
8. Abdullahi M, Shallangwa GA, Ibrahim MT et al (2018) QSAR studies on some C14-urea tetrandrine compounds as potent anticancer agents against leukemia cell line (K562). J Turk Chem Soc Sect A Chem 5(3):1387-1398. https://doi.org/10.18596/jotcs a. 457618

9. Isyaku Y, Uzairu A, Uba S (2020) Heliyon Computational studies of a series of 2-substituted phenyl-2-oxo-, 2-hydrox-yl- and 2 -acylloxyethylsulfonamides as potent anti-fungal agents. Heliyon 6:e03724. https://doi.org/10.1016/j.heliyon.2020.e03724

10. Attique SA, Hassan M, Usman M et al (2019) A molecular docking approach to evaluate the pharmacological properties of natural and synthetic treatment candidates for use against hypertension. Int J Environ Res Public Health 16(923):1-17. https://doi. org/10.3390/ijerph16060923

11. Becke AD (1993) Becke's three parameter hybrid method using the LYP correlation functional. J Chem Phys 98:5648-5652

12. Adeniji SE, Shallangwa GA, Arthur DE, Abdullahi M, Mahmoud AY, Haruna A (2020) Heliyon quantum modelling and molecular docking evaluation of some selected quinoline derivatives as antitubercular agents. Heliyon 6:e03639. https://doi.org/10.1016/j. heliyon.2020.e03639

13. Trott O, Olson AJ (2010) Autodock Vina: improving the speed and accuracy of docking with a new scoring function, efficient optimization and multithreading. J Comput Chem 22:455-461

14. Singh SP, Konwar BK (2012) Molecular docking studies of quercetin and its analogues against human inducible nitric oxide synthase. SpringerPlus 1:69

15. Bello A, Adamu U, Gideon U, Shallangwa A, Uba S (2020) Design of potential anti-melanoma agents against SK-MEL-5 cell line using QSAR modeling and molecular docking methods. SN Appl Sci. https://doi.org/10.1007/s42452-020-2620-8

16. Elijah S, David A, Arthur E, Abdullahi M, Haruna A (2020) Quantitative structure-activity relationship model, molecular docking simulation and computational design of some novel compounds against DNA gyrase receptor. Chem Afr. https://doi.org/10.1007/ s42250-020-00132-9

17. Daina A, Michielin O, Zoete V (2017) SwissADME : a free web tool to evaluate pharmacokinetics, drug-likeness and medicinal chemistry friendliness of small molecules. Nat Publ Group. https ://doi.org/10.1038/srep42717

18. Daina A, Zoete V (2016) A boiled-egg to predict gastrointestinal absorption and brain penetration of small molecules 1117-1121. https://doi.org/10.1002/cmdc.201600182 